\title{
Germanica
}

\section{La satire de la société wilhelminienne}

dans Solneman der Unsichtbare d'Alexander Moritz Frey

Die Satire der wilhelminischen Gesellschaft in Solneman der Unsichtbare von Alexander Moritz Frey

\section{Pierre Giraud}

\section{OpenEdition}

\section{Journals}

\section{Édition électronique}

URL : http://journals.openedition.org/germanica/2828

DOI : 10.4000/germanica.2828

ISSN : 2107-0784

\section{Éditeur}

Université de Lille

\section{Édition imprimée}

Date de publication : 30 juin 1988

Pagination : 169 à 184

ISSN : 0984-2632

\section{Référence électronique}

Pierre Giraud, «La satire de la société wilhelminienne », Germanica [En ligne], 3 | 1988, mis en ligne le 11 septembre 2015, consulté le 06 octobre 2020. URL : http://journals.openedition.org/germanica/ 2828 ; DOI : https://doi.org/10.4000/germanica.2828

Ce document a été généré automatiquement le 6 octobre 2020.

(c) Tous droits réservés 


\title{
La satire de la société wilhelminienne
}

\author{
dans Solneman der Unsichtbare d'Alexander Moritz Frey \\ Die Satire der wilhelminischen Gesellschaft in Solneman der Unsichtbare von \\ Alexander Moritz Frey
}

Pierre Giraud

\section{NOTE DE L'ÉDITEUR}

Les indications de pages mentionnées entre parenthèses dans le corps de l'article renvoient à l'édition de Solneman der Unsichtbare publiée chez Suhrkamp en 1984.

1 Des travaux universitaires récents, notamment ceux de Katrin Hoffmann-Walbeck ${ }^{1}$ et de Jean-Jacques Pollet $^{2}$ ont attiré il nouveau l'attention sur les œuvres injustement oubliées d'Alexander Moritz Frey, en particulier sur son meilleur roman Solneman der Unsichtbare ${ }^{3}$ paru en 1914 et réédité chez Suhrkamp en 1984. Un ouvrage dont l'intérêt n'avait échappé ni à Thomas Mann ${ }^{4}$, qui avait remarqué Frey dès 1909 et le soutiendra toujours, y compris matériellement, lors de son précaire exil en Suisse à partir de 1933, ni à Kurt Tucholsky 5 lors de la réédition de 1920.

2 Auteur de 24 livres parus entre 1913 et 1957 (essentiellement des recueils de nouvelles et des romans), Frey est généralement rangé parmi les écrivains fantastiques qui connaissent une grande faveur en Allemagne et en Autriche durant le premier quart du vingtième siècle.

3 Sa manière de traiter le fantastique est cependant un peu particulière. Lui-même a souligné plusieurs fois que les éléments surnaturels inscrits dans la trame de ses œuvres avaient pour fonction première de donner une vision critique du monde réel contemporain. Dans le «Curriculum vitae » cité par Katrin Hoffmann-Walbeck ${ }^{6}$ que Frey rédige en 1939 pour une demande d'émigration aux États-Unis (elle n'aura pas de suites), il écrit que ses récits et nouvelles ont «un contenu satirique, fantastique, 
grotesque et réel et qu'ils s'occupent de notre temps, de nos contemporains et de notre vie quotidienne $»$.

4 Le roman Solneman der Unsichtbare fournit une illustration significative de cette ambition dans la mesure où le déroulement des événements permet à l'auteur de présenter un tableau satirique de la société wilhelminienne. Cette critique se déroule à trois niveaux, d'abord à travers une galerie de personnages, ensuite à travers la figure de Guillaume II, enfin à travers la dénonciation d'une mentalité.

5 Notons au préalable que la structure du roman se prête particulièrement bien à la mise en place d'une visée satirique. Un inconnu d'apparence excentrique, Solneman, dont le nom est l'anagramme de «namenlos» (sans nom), fait irruption un beau matin dans le bureau du bourgmestre d'une ville d'Allemagne du sud qui, sans être désignée, ressemble fort à Munich. Contre une fabuleuse somme d'argent il lui propose de racheter le parc municipal où il souhaite s'isoler complètement pour se livrer à diverses expériences et surtout vivre pour lui-même. S'il s'engage à respecter l'intégrité du parc, il exige de ne jamais être dérangé par les habitants de la cité. Il aspire à une retraite absolue. C'est pourquoi il élèvera tout autour du parc une immense muraille de 30 mètres de hauteur qui le mettra à l'abri de tous les regards. Après quelques réticences de la part des édiles, le marché est finalement conclu et on devine que la suite du roman, distribuée en une série vivement agencée de brèves séquences, sera faite des tentatives multiples et toujours vaines de la population pour percer le secret de Solneman et des "horreurs » auxquels il est censé se livrer à l'intérieur de son enclave. On essaiera d'escalader le mur d'enceinte, de percer un souterrain, de survoler le parc en avion, de passer par les canalisations de la rivière souterraine, d'attirer Solneman hors de son repaire par divers subterfuges. Peine perdue. Toutes ces expéditions tournent à la confusion de leurs instigateurs jusqu'au jour où Solneman, excédé d'avoir perpétuellement à déjouer ces incursions, quitte définitivement le parc et la ville, non sans lancer toutefois une ultime menace.

Ce roman, fait d'une succession d'épisodes parallèles, dont la fin reste ouverte, repose donc sur l'opposition de deux univers ou, pour être plus précis, de deux sphères à l'intérieur du même univers, d'un côté celle des habitants de la ville de l'autre celle de Solneman retranché dans sa retraite. Par sa présence d'autant plus obsédante pour les citadins que nul d'entre eux n'a jamais vu son visage puisqu'il porte toujours un masque et que tous ignorent ses activités jugées attentatoires à l'ordre et à la morale, Solneman les pousse, comme le dit Jean-Jacques Pollet, à «fantasmer» et à délirer à partir de leur propre nature, de leur identité sociale et de leurs préjugés. Ils apparaissent alors pour ce qu'ils sont, sous leur vrai jour et avec eux donc la société qui est la leur. Solneman agit comme un révélateur. L'homme masqué les démasque. Joachim Manfred Wolff a tout à fait raison de faire un parallèle avec le divan du psychanalyste. Par ses prétendues provocations, Solneman est l'agent de la satire.

7 Cette opposition entre l'homme invisible et la foule en délire est donnée dès le début du roman lors de la première entrevue avec le bourgmestre. En lisant la lettre de Solneman, le premier magistrat a l'impression que "quelque chose d'énigmatique émanait de l'encre violette » et que "de visage et l'atmosphère d'un monde inconnu semblaient s'y trouver cachés» (page 6). Peu de temps après, Solneman venu lui remettre un gros diamant destiné à le convaincre du sérieux de ses intentions, ajoute malicieusement en riant : «Je vous remets ce gros caillou sans la moindre réticence, sachant bien que vous êtes un homme d'honneur; votre position vous interdit de toute 
façon d'être autre chose» (page 7). Dès lors le désir de Solneman de se couper du monde traduit d'emblée une distanciation critique qui permet à l'auteur d'instruire en toute complicité le procès de la société wilhelminienne. Tout d'abord à travers une galerie de personnages.

\section{Galerie de personnages}

8 Par sa tonalité et les moyens qu'elle se donne, cette satire reste toujours enrobée de fantaisie et de malice. Les personnages qui s'agitent dans cet univers sont très marqués, voire stéréotypés. Ils sont proches des silhouettes de la caricature. Frey sait les croquer d'un trait vif à la manière des dessinateurs du «Simplicissimus» où il a d'ailleurs publié plusieurs de ses récits. Le nom, à lui seul, suffit à camper les principaux protagonistes : le professeur Lautenschlag, le bourgmestre Bock et sa fille Claire dont le prénom ne reflète pas exactement la nature des intentions, l'artiste de music-hall Sirene Golfström, une plongeuse de haut vol qui essaie de s'introduire à la nage dans le domaine de Solneman, l'officier d'aviation von Eckern-Beckenbruch dont les périples aéronautiques se terminent toujours piteusement, le bedonnant conseiller juridique Schlicksupp qui a toujours un morceau de fromage dans ses poches et ne peut progresser sur l'échelle dressée contre le mur d'enceinte qu'avec l'aide de deux soldats, le dos tourné vers les barreaux. Satire visuelle, très évocatrice qui resterait cependant superficielle si les personnages n'étaient pas inscrits dans une réalité sociale aisément identifiable.

Ces personnages en effet sont plus que des marionnettes. Ils sont bien de leur temps dans un pays bien défini. Tout l'art de Frey consiste à suggérer que leurs comportements sont marqués de manière irrémédiable par le culte de l'autorité et la servilité propres au régime wilhelminien. Ce sont des "sujets" à la manière du "Sujet» de Heinrich Mann dont Frey était aussi l'ami et dont le roman, publié seulement après la guerre en 1918, fut néanmoins achevé comme "Solneman » en 1914 et commença d'être publié précisément à cette date dans la revue Zeit im Bild ${ }^{7}$.

S'ils ont dans l'appareil social des fonctions bien différenciées, ces petits-bourgeois ont abdiqué toute individualité et toute personnalité. Ils sortent tous du même moule, se renvoient et renvoient aux autres la même image. Ils sont parfaitement interchangeables, moutons innombrables du troupeau anonyme. L'anonymat n'est pas le seul fait de Solneman. Aux habitants qui l'interrogent fébrilement sur l'apparence de l'homme masqué (A-t-il des dents ? Porte-t-il une barbe ? Comment sont ses vêtements ?), l'ouvrier engagé par ce dernier pour effectuer des travaux dans le parc n'a pas grand chose à révéler faute de termes de comparaison adéquats, car, ajoute-t-il de manière très éclairante : «Vous vous ressemblez tous » (page 39).

11 Nombre d'entre eux sont affligés de tics de comportement parfaitement révélateurs de leur appartenance à une classe bien définie et à son esprit de caste. Ainsi les officiers. Von Eckern Beckenbruch, qui voit son premier atterrissage forcé (provoqué en fait par une fausse panne de carburant) tourner à la déroute, n'a d'autre ressource que de dissimuler sa fureur derrière un triple masque de correction, tandis que Solneman, lui, reste d'une courtoisie exemplaire. Remplir les réservoirs d'un appareil est indigne d'un homme de son rang (page 61). Dans la même galerie on peut ranger le journaliste Arthur von $G$. que son rédacteur a dépêché auprès de Mademoiselle Golfstrom mystérieusement ressurgie à Saint-Petersbourg après son séjour auprès du «nabab ». 
Après une nuit fort prometteuse, où Golfström perce son jeu in extremis, il est tiré du sommeil sans ménagement par la belle plongeuse et si, au moment de la quitter, ses yeux sont encore à moitié endormis, ses talons, obéissant à une sorte de réflexe conditionné, ont encore la force de claquer magistralement comme il sied à un ancien officier (page 161).

L'égoïsme forcené et l'esprit de lucre qui animent toutes ces figures sont tout à fait représentatifs du matérialisme que l'on prête généralement à l'époque wilhelminienne. À cet égard on n'a que l'embarras du choix tellement les exemples sont nombreux et cet effet cumulatif, outre qu'il appartient à la panoplie traditionnelle du style satirique, vise sans doute aussi dans l'esprit de Frey à souligner que la société de l'époque est frappée d'un mal collectif, d'une sorte d'endémie.

Désintéressement et générosité ne sont pas les vertus dominantes des élites rassemblées dans la salle du conseil pour envisager la manière dont il faut investir les sommes énormes remises par Solneman. S'il est beaucoup question de construire des monuments à la gloire des impératrices (l'actuelle et la précédente) et du prince héritier récemment décédé, la part réservé aux chômeurs reste dérisoire (page 15).

Hannes Baum, directeur d 'une revue au service du matérialisme et du rationalisme, veut certes tout faire pour combattre une secte dévouée au culte de Solneman et en détourner les adeptes, mais ce n'est là à ses yeux qu'un objectif secondaire. L'essentiel pour lui est de faire augmenter le tirage de sa publication, notamment grâce à la parution d'une interview de Solneman. Les intentions de Claire Bock, la fille du bourgmestre, qui pense avoir réussi à faire une photo de l'étrange inconnu, appartiennent au même registre. La prétendue photo, accompagnée de la sienne, est vendue à tous les journaux de la ville et du pays, on en tire des cartes postales et comme Claire Bock a un pourcentage sur les ventes, elle ne tarde pas à amasser une petite fortune (page 181).

ce domaine les conseillers municipaux ne le cèdent en rien à la fille de leur bourgmestre. La façon dont ils conçoivent la gestion de l'argent public est tout à fait éloquente, particulièrement dans l'épisode de l'immense tour métallique qu'ils font assembler et dresser par un ingénieur suisse pour pouvoir enfin espionner Solneman. Son prix n'est pas mince, mais cette considération ne saurait les faire reculer, car ils se disent qu'une fois qu'elle aura rempli son office, ils pourront toujours la revendre, de préférence à un organisme d'état (page 166).

16 Ce cynisme ouvertement affiché va de pair avec une insupportable vanité. Frey en donne une image saisissante renforcée par l'emploi du pluriel lorsqu'il décrit les délibérations du conseil municipal: «Durant les délibérations on se carrait sur des postérieurs imposants..., on arrondissait des bedaines sur des cuisses bouffies de graisse » (page 20).

17 Tous ces gens adorent le clinquant, la parade, comme le soulignent en particulier tous les épisodes consacrés aux préparatifs qui précèdent la venue de l'Empereur Guillaume II. La décoration de la ville est reprise de fond en comble. On recouvre les maisons de branchages. À tous les carrefours sont installés des arcs de triomphe. Les fabricants de drapeaux et de soutache s'enrichissent en un tournemain. Cette vénération ostentatoire s'empare même des objets requis pour cette mobilisation générale: d'immenses piedestals se dressent fièrement au milieu de l'agitation fébrile de la grande ville "avec la servilité qui convient aux sujets» (page 64). Cet amour du clinquant est inscrit dans leur psychè et lorsque Frey veut donner une idée des coqs qui 
composent la ménagerie de Solneman et dont le plumage bigarré éclate en gerbes étincelantes, il les compare naturellement au poitrail du bourgmestre revêtu de toutes ses décorations (page 43). Pour suggérer l'apparence d'un animal, Frey inverse l'ordre habituel des termes de la comparaison, l'homme servant ici de référent.

Ces bons sujets sont fiers de l'être et ne craignent pas de le signifier avec une conscience hypertrophiée de leur mission. L'excès de zèle est chez eux une seconde nature. Ainsi l'agent de police 216 qui, cherchant à interpeller une Vénus nocturne ayant quelque ressemblance avec la négresse de Solneman, va au-delà de ce que lui imposent ses obligations de service et poursuit ses investigations clans le secteur imparti à l'un de ses collègues (page 189).

Ces spécimens de l'Allemagne wilhelminienne se renieraient s'ils ne communiaient pas dans la célébration immodérée de la technique et des moyens d'action qu'elle procure. On reconnaît bien là une idolâtrie de l'époque et Frey ne se fait pas faute de la tourner en dérision, ne serait-ce qu'en montrant systématiquement ses limites ou ses échecs toujours imprévus. Les jeunes officiers qui envisagent de survoler en dirigeable le parc de Solneman ont programmé tous les risques. Pour déjouer les balles éventuelles qui leur seraient destinées, ils ont conçu un énorme pulvérisateur qui diffusera un épais nuage et dont le brevet a d'ores et déjà été déposé auprès de l'office compétent du Reich. Comme tant d'autres et malgré cette géniale invention, cette tentative tournera court. L'épisode de la tour évoqué ci-dessus illustre plus nettement encore le même thème. L'ingénieur suisse qui l'a conçue est ulcéré de voir que Solneman, légitimement inquiet de ce qui se prépare, puisse le confondre avec ce «célèbre comte allemand qu'un ordre suprême de Sa Majesté a consacré comme le plus grand homme du vingtième siècle » (page 165), ce qui est une allusion transparente et sarcastique au Comte Zeppelin et au culte dont il faisait alors l'objet.

L'ascension de cette tour fournit d'ailleurs au bourgmestre une occasion d'exalter audelà de toute mesure le sens de sa mission. Elle donne lieu de sa part à une envolée épique. Convaincu d'être à la veille de réduire enfin Solneman, il escalade les tubulures métalliques avec le sentiment hypertrophié d'être le champion symbolique d'une grande cause, « la lumière qui triomphe des ténèbres... une sorte d'incarnation de cette bonne cause qui oppose sa face d'éternité à la méchanceté de l'ennemi» (page 167). Prétention d'autant plus ridicule que, quelques minutes plus tard, le bourgmestre va se voir contraint d'amorcer une piètre retraite.

21 Tics de comportement, égoïsme, matérialisme, servilité, idolâtrie de la technique sont autant de signes qui nous permettent d'identifier sans équivoque des sujets de Sa Majesté Guillaume II. Mais Frey ne s'en tient pas là. Il va plus loin. Sa Majesté en personne est présente dans le roman, prêtant même son titre à l'un des chapitres. Avec Des Untertan de Heinrich Mann, Solneman der Unsichtbare est ainsi l'un des rares romans de la littérature allemande à présenter directement Guillaume II à l'époque même de son règne pour donner de sa personne et de son idéologie une vision fort irrespectueuse.

\section{La figure de Guillaume II}

22 Frey en effet ne se contente pas de brocarder les manifestations grotesques auxquelles donnent lieu chez les sujets de l'Empereur le conformisme le plus servile. En 
introduisant l'Empereur dans le cours de l'action, il nous livre la clef de ce mimétisme. Ces bons citoyens ne font que singer leur maître, leur suprême référence.

Dans la présentation qu'en donne le romancier, cette référence aisément reconnaissable a tout du modèle négatif. Si ses sujets sacrifient spontanément au goût de la parade, c'est que lui-même est sans cesse en représentation et situe à ce niveau l'essentiel de sa fonction. Parodiant le style homérique, Frey le désigne comme celui qui fait cliqueter ses éperons au moindre signe d'impatience («Der Sporenklirrende» page 69). Si l'Empereur - tout comme la ville de Munich - n'est pas désigné nommément dans le roman, il est aisément reconnaissable à ces quelques détails qui déjà frappaient les contemporains. D'autres indications sont tout aussi explicites, ainsi l'allusion aux attentats dont il avait failli être victime au début de son règne. L'Empereur ayant manifesté le souhait de rencontrer Solneman ou plus exactement de pouvoir le dévisager de loin (sa bravoure a tout de même des limites), le maire en éprouve une grande perplexité et se demande si cette requête est bien raisonnable, car derrière Solneman, qu'après tout nul ne connait, pourrait se cacher un poseur de bombes ou un dangereux anarchiste (page 68). Mais cette crainte est vite balayée, car, ajoute-t-il en bon serviteur respectueux des dogmes, nous sommes tous placés sous la protection de Dieu et à fortiori celui qui a été élu par Dieu.

Les raisons pour lesquelles Sa Majesté tient tellement à rencontrer l'ermite du parc n'ont d'ailleurs rien de désintéressé. Bien au contraire. Elles s'expliquent avant tout par le parti qui pourrait être tiré de ses immenses richesses. Elles permettraient en effet de contracter auprès de lui des emprunts assez colossaux, voire très colossaux (page 69). Et lorsque, peu auparavant, le bourgmestre confie à l'Empereur que Solneman a pour habitude de régler ses dettes argent comptant, Sa Majesté y voit une «faiblesse plaisante » de sa part (page 70).

Cynisme donc, mais aussi aveuglement par excès de présomption, car Solneman, lui, est tout sauf naïf, et représente de toute façon l'anti-sujet par excellence. Il est en effet piquant de constater que c'est précisément le caractère envahissant de l'idéologie impériale qui fera échouer l'entrevue entre Solneman et l'Empereur. Les autorités de la ville ont imaginé d'attirer Solneman à la perception sous un fallacieux prétexte. Mais à peine celui-ci a-t-il franchi le seuil de cette administration que son attention est immédiatement frappée par une débauche de pancartes apposées au-dessus des portes qui, toutes, délivrent, sous forme de devises martiales, un message agressif parodiant sans ambiguïé l'impérialisme wilhelminien : "Fidélité à toute épreuve ", « Nunquam retrorsum ", "Quiconque a confiance en Dieu et distribue sans relâche des coups à la cantonade, ne bâtit par sur du sable ». Il n'en faudra pas plus pour réveiller la méfiance de Solneman et lui faire rebrousser aussitôt chemin. L'affichage ostensible de l'autoritarisme tourne à la déroute de son inspirateur et de ses prosélytes (page 71).

Il existe d'autres signes plus révélateurs encore de cette volonté de sujétion à quoi se résume finalement l'esprit wilhelminien. On les repère au niveau du discours et du langage que Frey met dans la bouche de ses personnages. Un langage ampoulé, déclamatoire, empreint de lourdeur bureaucratique qui ne fait que refléter l'éloquence théâtrale de Sa Majesté et constitue une marque d'allégeance supplémentaire. "On savait que Sa Majesté aimait le clinquant et les discours approbateurs qui tombaient de sa très auguste bouche indiquaient à quel point Sa Majesté l'aimait » (page 69). Par mimétisme tous les personnages adoptent ce mode d'expression dont Frey renforce l'effet parodique en rapportant, la plu part du temps, leurs propos au discours indirect. 
Le recours à ce mode décalé, s'il fait ressortir l'abdication de toute spontanéité et de tout naturel, accentue également le contraste entre la recherche alambiquée de l'expression et la trivialité des intentions qui, toutes, tournent peu ou prou autour d'objectifs purement mercantiles. Depuis le joaillier, qui rédige son expertise sur le diamant donné en gage par Solneman au début du roman dans le plus pur style administratif, en passant par Claire Bock, dont les divagations sentimentales amènent Solneman à lui demander si elle a fait des études de philosophie, jusqu'à son père, le bourgmestre, tous sacrifient au style de chancellerie qu'ils ont parfaitement intériorisé. La portée satirique est d'autant plus réussie que, par contraste, Solneman dans les rares messages qu'il adresse aux citadins ou à leurs représentants opte toujours pour un style laconique, elliptique même, proche du style télégraphique: "Halte là ", s'écria l'étranger, " aucune information, à personne et sur quoi que ce soit. Par contre ceci de bon gré : les arbres, les cours d'eau resteront intacts. Suis libre de construire des édifices... » (page 11).

Par ce biais, mais aussi par d'autres moyens, Solneman ne se fait pas faute de leur renvoyer l'image de leur véritable nature, de ce qu'ils sont c'est-à-dire des singes de l'Empereur. Est-ce un hasard si, lors de l'incursion finale dans le parc, les envahisseurs emmenés par le bourgmestre retrouvent le lieutenant von EckernBeckenbruch enfermé, après sa deuxième tentative d'atterrissage, nu dans une cage au milieu des singes? Ses sauveteurs n'estiment d'ailleurs n'avoir rien de plus urgent à faire que de lui procurer l'accessoire qui consacrera son retour dans la communauté des hommes: un uniforme!

Solneman lui-même joue à merveille des réflexes conditionnés de ces marionnettes impériales. Quand il lance à toute allure ses canaux à moteur sur le plan d'eau aménagé au sommet du mur d'enceinte, il veille à ce que la succession de leurs couleurs reproduise exactement celle du drapeau impérial : noir, blanc et rouge, ce qui déchaîne aussitôt les vivats de la foule de badauds et lui vaut quelques sympathies momentanées (page 109). Le jeune exalté qui, des fenêtres de son appartement, veut abattre Solneman d'un coup de canon, mais ne réussit qu'à ouvrir une brèche dans la muraille, provoquant ainsi l'inondation de la ville, ne laisse planer aucune équivoque sur ses motivations. Après cette tentative d'attentat, il se jette dans le vide en hurlant la fameuse devise : «Avec Dieu, pour le roi et la patrie » (page 196).

La dénonciation sur le mode grotesque du nationalisme à travers son incarnation suprême et ses mimes anonymes constitue l'une des composantes majeures du roman et court tout au long des pages. Elle se double d'une satire discrète de ce qu'on pourrait appeler un égocentrisme culturel.

\section{La dénonciation d'une mentalité}

Elle touche d'abord au domaine de l'art. En matière esthétique, tous ces philistins ont des conceptions tout à fait conformistes et étriquées. Ils ont un faible pour les monuments commémoratifs, surtout s'ils sont pompeux. La seule peinture qui leur parle est la peinture platement réaliste et figurative. Les tableaux gigantesques que Solneman a fait exécuter sur toute la surface du mur d'enceinte les déroutent. Un tel croit $\mathrm{y}$ déchiffrer une tête de garçon, un autre un harmonica, un troisième un $\mathrm{n} \mathrm{u}$ féminin (page 30). 

ils ne tardent pas à découvrir une statuette votive en bois que Solneman a dressée dans le temple grec à la place de la statue du fondateur et qui est une figuration emblématique du cubisme inspiré de la statuaire nègre. Belle occasion pour le narrateur, à travers les commentaires qu'en donnent ces petits-bourgeois, de fustiger leur rejet absolu de l'esthétique moderne. Non seulement ils y voient comme le premier procureur (qui est aussi à ses heures un peintre du dimanche) le comble de la laideur, «une association de contrastes absolument incompatibles» (page 211), «une juxtaposition insensée d'éléments qui ne peuvent se conjuguer que dans les affres » (page 212), mais aussi le signe manifeste d'une perversion susceptible de contaminer l'âme collective. L'art moderne est jugé à la fois décadent et dissolvant. Outre que cette condamnation absolue reflète fidèlement l'opinion des milieux officiels de l'époque, elle prend aussi à deux décennies de distance une résonnance fâcheusement prophétique.

Tous ces braves petits-bourgeois sont la proie d'un délire. Cette statuette leur paraît cacher «une vie qui restait muette, mais qui, sans doute, continuait de palpiter sous cet épiderme foncé - là, à portée de la main, prête à bondir. Si on s'attardait à la contempler, elle pouvait bien vous sauter à la gorge et étouffer sa victime au milieu des cris perçants rappelant la cacophonie des forêts tropicales et le fracas des grands fleuves exotiques » (page 210).

La référence à la « cacophonie des forêts tropicales » ne tombe pas par hasard et n'est pas innocente. Elle est le signe d'une prétendue supériorité culturelle dont les connotations raciales, bien qu'indirectement suggérées, n'en sont pas moins nettement perceptibles.

34 Elles apparaissent aussi dans les chapitres consacrés à la réception de la princesse Santu-Bibi dont l'auteur laisse entendre, comme en bien d'autres occasions, que la venue a été manigancée par Solneman. Elle sert en tout cas de révélateur. Si les autorités de la ville décident de le recevoir avec tous les honneurs dûs à son rang, c'est bien sûr d'abord par calcul, car, prenant conscience de l'affaiblissement des nations européennes, ils jugent plus prudent de ménager l'avenir, mais c'est surtout parce qu'ils sont flattés de voir la fille du roi du Congo ainsi que ses 23 frères venir goûter dans leurs universités «la sagesse des vieilles cultures vivifiée par les feux d'une nouvelle aurore » (page 83). Ils se jugent culturellement parlant très supérieurs à ces sauvages. Supériorité que démentira le déroulement de l'épisode. Tout d'abord, ils s'avèrent incapables de distinguer la vraie princesse d'une fausse princesse arrivée inopinément la veille que le bourgmestre essaie en vain d'amadouer avec des colifichets (ses propres décorations) conformément aux préjugés qu'on lui a enseignés à l'école.

Dans ce procès instruit contre la supériorité de la culture germanique le narrateur multiplie les insolences et les sarcasmes. Si la vraie princesse est accompagnée de deux préceptrices chargés de lui enseigner les langues, aucune n'est allemande, car, comme le dit le directeur de l'hôtel: "L'allemand n'est pas une langue qu'on puisse se permettre d'utiliser devant des éminences étrangères » (page 210). D'ailleurs lorsqu'on veut expliquer au maire ce que sont et à quoi ressemblent des plumes de marabout qui permettront d'identifier la princesse parmi ses dames de compagnie, le dictionnaire, en l'occurrence le fameux «Brockhaus», est de peu de secours et après l'avoir consulté, le directeur de l'hôtel rassure le bourgmestre en lui disant qu'il lui suffira de songer à du duvet d'aigle dans sa version africaine (page 185). 

des silhouettes à la Wilhelm Busch, de ne pas s'attaquer à la dénonciation des structures sociales et des inégalités caractéristiques de la société d'alors. De telles affirmations, sans être totalement infondées, reviennent à oublier que la visée de Frey n'est pas celle du réalisme. Son propos est de faire par l'entremise d'un personnage étranger au réel le procès d'une mentalité, d'une attitude culturelle où l'autoritarisme et la servilité renvoient l'une à l'autre. Il se rattache étroitement au sujet central de l'œuvre qui, comme le dit Jean-Jacques Pollet', est «le rejet de l'autre et de la différence ». En marge du fantastique s'ouvrent ainsi des perspectives troublantes que l'évolution historique de l'Allemagne viendra, hélas, confirmer. Il ne s'agit pas de faire de Frey une sorte de prophète, mais force est bien de reconnaître que sa plume endiablée a su mettre à nu des symptômes peu rassurants. Cette dimension du roman, souvent insoupçonnée à l'époque, n'a pas échappé à un observateur aussi avisé que Kurt Tucholsky qui, dans son compte rendu de «Die Weltbühne», note " qu'il y a en outre (dans ce roman) un ton qui fait dresser l'oreille et qui n'est pas né dans le champ de coquelicots d'E.A. Poe : un ton tranchant et glacial $^{9}{ }^{\prime}$.

Dans un article récent, Peter Cersowsky ${ }^{10}$, examinant la question de savoir si le fantastique politique n'est pas une contradiction en soi, aboutit à la conclusion que les œuvres des auteurs fantastiques allemands du début du siècle relèvent d'une esthétique conservatrice, voire réactionnaire. Elles préfigureraient à leur manière peu ou prou le national-socialisme - « ni moins, mais pas plus non plus que le conservatisme d'un Moeller van den Bruck ${ }^{11}$ » qui, comme on le sait, a été l'introducteur de Poe en Allemagne. Pour ce faire, il s'appuie essentiellement sur les œuvres d'Ewers, de Meyrink et de Frey qui, toutes, sacrifieraient à une conception organiciste de la vie et enseigneraient la soumission à la fatalité du destin. La démonstration paraît injuste pour Alexander Moritz Frey. Le fait que seul son roman pacifiste Die Pflasterkästen ${ }^{12}$, 
paru en 1929, ait figuré sur les listes noires du régime nazi et non ses autres œuvres ne suffit pas à rendre la démonstration entièrement convaincante. Les censeurs n'ont pas toujours eu, Dieu merci, un flair infaillible pour la subversion.

41 En faisant fonctionner le fantastique comme révélateur de certaines dérives particulières à son temps et en montrant en acte sur le mode grotesque les aberrations d'une psychose collective, Frey prolonge une filiation déjà présente dans l'œuvre d'E.T.A. Hoffmann et place son roman Solneman der Unsichtbare sous le signe de la tolérance et du pacifisme humaniste. Preuve, s'il en est, que le fantastique, loin de servir de vecteur à la fuite hors du monde vers un ailleurs consolateur, peut se faire l'instrument d'une critique de la société.

\section{NOTES}

1. Katrin Hoffmann-Walbeck, Alexander Moritz Frey (allegorische) Phantastik und Groteske als Mittel zur Zeitkritik, Peter Lang, Inaugural-Dissertation, 1984. Pour Solneman der Unsichtbare voir plus spécialement pp. 143-181.

2. Jean-Jacques Pollet, Essai sur la littérature fantastique allemande du début du XXe siècle (1900-1930), doctorat d'État, Université de Paris X-Nanterre, Publications de l'Université de Lille III, 1985. Pour Solneman der Unsichtbare voir plus spécialement pp. 340-344.

3. Alexander Moritz Frey, Solneman der Unsichtbare, Suhrkamp, 1984. La traduction française de ce roman par Jean-Jacques Pollet et Pierre Giraud doit être publiée prochainement chez Christian Bourgois sous le titre « Mon nom est personne".

4. Thomas Mann, Liebenswerte Menagerie, 1955, Das essayistische Werk, Taschenbuchausgabe in 8 Bünden, Frankfurt/Main, Mk120, pp. 255-256, cité d'après Jean-Jacques Pollet, op. cit., p. 340.

5. Kurt Tucholsky (Peter Panter), «Solneman der Unsichtbare», in Die Weltbühne 15, Jg. Nr 33, 7/8/1919, S. 177-178.

6. Joachim Manfred Wolff, «Alexander Moritz Frey, Solneman der Unsichtbar», s. 165-175, in Spiege/ irn dunklen Wort, Analysen zur Prosa des frühen XX, Jahrhunderts, Herausgeber: Winfried Freund, Hans Schumacher, Peter Lang, 1983.

7. Wolfgang Emmerich, Heinrich Mann, Der Untertan, Fink Verlag, 1980.

8. Jean-Jacques Pollet, op. cit., p. 344.

9. Peter Tucholsky, op. cit., p. 178.

10. Peter Cersowsky, «Ja, mein Lieber, wir sind konservativ», Politische Aspekte bei deutschsprachigen Phantastikautoren des XX, Jahrhunderts bis zum Nationalsozialismus, in Die dunkle Seite der Wirklichkeit, Suhrkamp, 1987, pp. 33-58.

11. Peter Cersowsky, op. cit., p. 54.

12. A.M. Frey, Die Pflasterkästen, ein Feldsanitätsroman, Gustav Kiepenheuer Verlag, 1929 - réédité en R.D.A., 1984, Gustav Kiepenheuer Verlag, Leipzig und Weimar et en 1986 en R.F.A., Fischer Taschenbuch Verlag, verboten une verbrannt/Exil. Dans cette œuvre autobiographique qui se déroule pour l'essentiel sur le fronl allemand du Nord de la France, Frey relate sans emphase, avec un grand souci de vérité, ses expériences d'infirmier et les réalités cruelles de la guerre. À l'époque, ce roman fut largement commenté par la critique et souleva beaucoup de polémiques. Cf. entre autres trois articles publiés dans Die Weltbühne, Karl Hugo Sclutius, pazifistische Kriegspropaganda in Die Weltbühne, $25 \mathrm{Jg}, 2 / 4 / 1929$, Nr 14, S. 517-522. Arnold Zweig, 
«Kriegsromane», in Die Weltbühne, 25. Jg, 16/4/1929. Nrl6, S.597-599. Carl von Ossietzky, «Die Pflasterkästen», in Die Weltbühne, 25. Jg., 30/4/1929, Nr 18, 2. 686-587.

Tous les trois placent cette œuvre très au dessus d'«Im Westen Nichts Neues» d'Erich Maria Remarque et de «Krieg» de Ludwig Renn (parus peu auparavant) dans la mesure où son contenu, dépourvu de toute ambiguité et de toute hérö̈sation, ne saurait servir de caution aux propagandes bellicistes. En 1927, Die Welt bühne avait publié en pré-publication un extrait du roman tout à fait révélateur de la manière de Frey : «Die Maus und die Granate», in Die Weltbühne, 23. Jg, 7/6/1927, n²3, S. 905-908.

À noter enfin que Frey a servi durant la première guerre mondiale dans le même régiment que Hitler sur lequel il a laissé un témoignage non publié et non daté : «Der unbekannte Gefreite» «Persönliche Erinnerungen an Hitler», Nachlass, Schiller Nationalmuseum, cité d'après K. Hoffmann-Walbeck, op. cit., S. 82.

Sollicité par les nazis pour apporter son soutien au nouveau régime, Frey leur opposera un refus catégorique et immédiat et devra quitter précipitamment l'Allemagne.

\section{RÉSUMÉS}

Grâce à divers travaux universitaires parus en Allemagne et en France on redécouvre les œuvres de l'écrivain fantastique A.M. Frey et en particulier son roman Solneman der Unsichtbare (1914), dont l'importance n'avait pas échappé alors à Thomas Mann et à Kurt Tucholsky. Frey a toujours souligné qu'à ses yeux le fantastique, loin d'inciter à une fuite hors du monde, avait une fonction satirique et critique et s'occupait de l'époque, des contemporains et de la vie quotidienne. Solneman der Unsichtbare illustre de manière convaincante cette ambition dans la mesure où l'auteur réussit à dévoiler sur le mode grotesque et humoristique certaines défaillances et certains éléments « inquiétants » de la société wilhelminienne.

Dank verschiedener wissenschaftlicher Arbeiten in Deutschland und Frankreich werden die Werke des phantastischen Schriftstellers A.M. Frey wieder entdeckt, insbesondere sein Roman Solneman der Unsichtbare (1914), dessen Bedeutung Thomas Mann und Kurt Tucholsky damals nicht entgangen war. Frey hat immer wieder darauf hingewiesen, dass in seinen Augen das Phantastische, weit davon ent fernt, zur Flucht aus der Welt anzuregen, eine kritisch-satirische Funktion besitze und sich mit der Zeit, den Zeitgenossen und dem Alltag bcschaftige. Solneman der Unsichtbare veranschaulicht überzeugend diesen Anspruch, insofern es dem Autor gelingt, auf grotskc und humorvolle Weise gewisse Mtingel und gewisse «beunruhigende» Elemente der wilhelminischen Gesellschaft aufzudecken.

\section{AUTEUR}

\section{PIERRE GIRAUD}

Université de Lille III 